\title{
MONACO v2: Multipurpose and Integrated Data Acquisition System for On-line Neutron and Gamma Measurements
}

\author{
L. Barbot, D. Fourmentel, G. De Izarra, C. Destouches, J-F. Villard, Y. Moline and H. Hamrita.
}

\begin{abstract}
The CEA MONACO v2 a multichannel acquisition system dedicated to neutron and gamma measurements. It is unique as it integrates all the following features in one module: automatic generation of saturation curves, automatic generation of pulse discrimination curves, detector pulse characterization using the embedded oscilloscope module, pulse mode acquisitions in count rate or pulse height analysis, fluctuation mode and current mode acquisitions. Sensors are plugged to a single connector and the implemented operating modes run constantly in parallel.

Firsly designed for on line local neutron and gamma measurements with gaseous detectors in reactor experiments, the MONACO v2 system will also be available for self-powered detectors thanks to its wide current mode working range.

After two years of development, CEA teams tested two MONACO v2 prototypes in the Slovenian TRIGA Mark II research reactor in 2018, using CEA miniature ion chambers and SPNDs. The system is now ready for industrialization to be available on the nuclear instrumentation market.
\end{abstract}

Index Terms-Neutron instrumentation, Neutron acquisition system, Gaseous detectors, Ion chamber, Fission chamber, Selfpowered neutron detectors, SPND, FPGA, Research reactor.

\section{INTRODUCTION}

CONSIDERING local neutron flux measurement techniques in

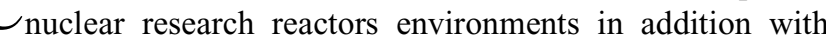
reactor command control, online gaseous detectors are suffering from the lack of up-to-date and integrated signal acquisition system on the nuclear instrumentation market. CEA needs for research reactor neutron field characterization led to the development of a multipurpose and integrated data acquisition system for online neutron and gamma measurements called MONACO, standing for 'Multichannel Online Neutron Acquisition in Campbell mOde'. After two years of development, this paper presents the recent second version [1][2] features and the validation of two MONACO v2 prototypes in the Slovenian TRIGA Mark II reactor.

Manuscript received May $13^{\text {rd }}, 2019$. This work was supported by the Nuclear Instrumentation project (INSNU) of the CEA Nuclear Energy Division

L. Barbot, D. Fourmentel, G. De Izarra, C. Destouches and J-F. Villard are with the CEA, DEN, DER, Instrumentation, Sensors and Dosimetry Laboratory, Cadarache, F-13108 St-Paul-Lez-Durance, France (e-mail: loic.barbot@cea.fr).

Y. Moline and H. Hamrita are with the CEA, LIST, Sensors and Electronics Laboratory, Saclay, F-91191 Gif sur Yvette, France (e-mail: yoann.moline@cea.fr).

\section{CEA DEVELOPMENT}

\section{A. Context}

This CEA project aims at improving the neutron and gamma sensor signal processing during in-core neutron and gamma flux measurements in nuclear reactors. In this environment, two kinds of radiation detectors are commonly considered for online measurements: self-powered detectors and gaseous detectors. This development project is focusing particularly on the latter, where detectors are called 'ion chambers' for gamma flux measurements and 'fission chambers' for neutron flux measurements (a fissile coating on one electrode which renders it sensitive to neutrons). The intended improvement proposes to widen the range of operation of these detectors, facilitating their implementation and signal acquisition as well as the analysis of delivered signals.

During an axial and radial core mapping campaign, neutron flux levels are spread over several decades, meaning fission chambers operated successively in multiple modes. Operators, then, usually have to resort to a high number of different electronics to perform preliminary detector tests, detector calibrations and actual signal acquisitions.

\section{B. Neutron gaseous detector operation}

Under irradiation, neutrons induce emission of heavy fission products which ionize the filling gas (i.e. generate electron-ion pairs) between the two electrodes. The signal at the output of the detector results in current pulses.

Considering the neutron flux conditions in the core of nuclear reactors, fission chambers are ran in three different operating modes: pulse, current and fluctuation modes. Pulse mode is used at low to moderate neutron flux levels, where pulses (with typical width of a few tens of nanoseconds) are processed individually. At higher neutron flux, pulses may overlap up to the point of generating a mean current, which corresponds to the current mode. In the intermediate mode (fluctuation mode or Campbell mode [3]), where pulses are piling-up increasingly), the signal is assimilated to a noise superimposed to the mean current.

Because of this 'Poisson' noise, higher order moments of the signal are related to the neutron flux using the Campbell theorem. In our case, the second order moment (i.e. variance of the signal) is used to estimate the incident neutron flux. 
These modes allow such a detector to operate on a wide neutron flux range from $1.10^{+5}$ to $1.10^{+15}$ neutrons $\mathrm{cm}^{-2} \cdot \mathrm{s}^{-1}$. [4].

In addition to fission chamber, gamma ion chambers are processed using the single current mode.

\section{New acquisition system requirement}

This development tends to ease the implementation of ion and fission chambers as part of in-core instrumentation, gathering all required data acquisition systems in one, including a high voltage module for detector biasing, in order to cover a wide range of neutron flux levels.

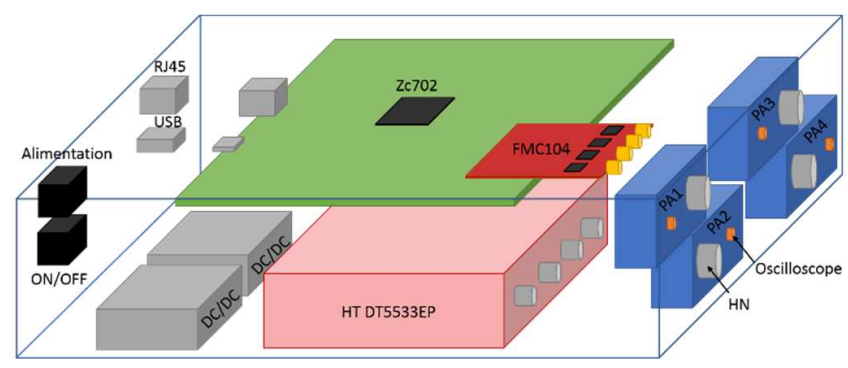

Figure 1: MONACO V2 3D synoptic [5] and 19" rack integration.

The CEA MONACO v2 system technical specifications starts from generic and global definitions to highly described specific features. It is a transportable four independent channel acquisition system with a high immunity to electromagnetic disturbances as well as required high reliability level and measurement reproducibility using a wide range of gaseous detectors with output signal varying in the $0-1 \mathrm{~mA}$ current range, $1 \mu \mathrm{A}-10 \mu \mathrm{A}$ pulse amplitudes and 20ns $-200 \mathrm{~ns}$ pulse widths. Each fission chambers is plugged to a single connector and the implemented operating modes run constantly in parallel.

The MONACO v2 system is unique as it integrates all the operator-required features in one module: four independent $0-+1000 \mathrm{~V}$ high voltage power supply, automatic generation of saturation curves, automatic generation of pulse discrimination curves. It includes a detector pulse characterization (mean amplitude and mean area) using the embedded oscilloscope module, the pulse mode acquisitions in count rate (MCS) is up to $1.10^{6} \mathrm{c} . \mathrm{s}^{-1}$ for axial profile measurements or in pulse height analysis (PHA) for detector calibration. The fluctuation mode is a continuous estimation of the signal variance over a configurable frequency range. Variances, in $\mathrm{A}^{-\mathrm{Hz}^{-2}}$, are supposed to cover a minimum of seven decades of neutrons flux. The current mode is introduced in this MONACO v2 system for acquisitions at high neutron flux levels or gamma measurements (ion chambers). Six current decades are required from $\ln A$ to $1 \mathrm{~mA}$.

For all operating modes, acquisition integration time is set to be variable over some specific values from $10 \mathrm{~ms}$ to $100 \mathrm{~s}$. This module is also unique as it makes accessible the analysis of the operating modes overlapping using a sole acquisition system
The CEA Instrumentation, Sensors and Dosimetry Lab (LDCI) designed features and required technical specifications whilst the CEA LIST Sensors and Electronics Lab (LCAE) developed the electronic board and associated signal processing.

\section{MONACO V2 SYSTEM DEFINITION}

The first two MONACO v2 prototypes are embedded in 19 inches racks.

The system is built around a FPGA Zc702 board from Xillinx. The four measurement channels are composed of current preamplifier, High Voltage supply and Analog to Digital Converters sampled at $250 \mathrm{MHz}$. The Digital Pulse Processing (DPP) architecture is implemented on the Zynq7020 System on Chip (SoC). The SoC integrates an FPGA part called Programmable Logic and a Dual-core ARM Cortex-A9 representing the Processing System.

Programmable Logic is here to extract all the required features of the signal at maximum frequency $(250 \mathrm{MHz})$ to maintain the data flow and avoid processing dead-time. It is based on fully configurable and reproducible DPP tiles associated to each channel. This tiles achieve a set of signal processing as band pass filtering, smoothing, Base Line Restoration, fast timing discrimination to extract all conventional DPP features associated to nuclear instrumentation (pulse height or width, counting, timestamp, pulse capture) and preprocessing of terms used in variance computation. Then, a fully scalable concentrator module bulks all the extracted features up in streamed frames.

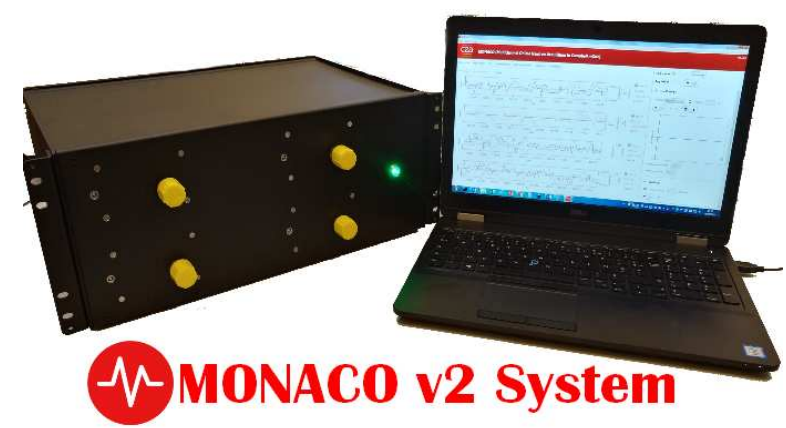

Figure 2: Photograph of the MONACO V2 system prototype

The resulting stream is accessible from the Processing System part with a Direct Memory Access module running at high throughput thanks to the DDR3 RAM. Number of tiles, size of frames, and timing constraints are completely independent of the Processing System part. The end-user supervision is developed via a high level Graphical User Interface. The overall architecture will be described in a dedicated full paper at this 2019 ANIMMA conference [5]. 


\section{RESEARCH REACTOR TESTS}

\section{A. Experimental Set-up}

\section{1) TRIGA Mark II research reactor}

The TRIGA Mark II reactor at the Jožef Stefan Institute (JSI - Ljubljana - Slovenia) is a pool type research reactor (250kW - first criticality in 1966) cooled by natural water convection. The uranium zirconium-hydride fuel mixture designed by General Atomics has unique properties that make the reactor inherently safe and suitable for training, research and isotope production. The reactor power is monitored with five independent excore detectors [6].

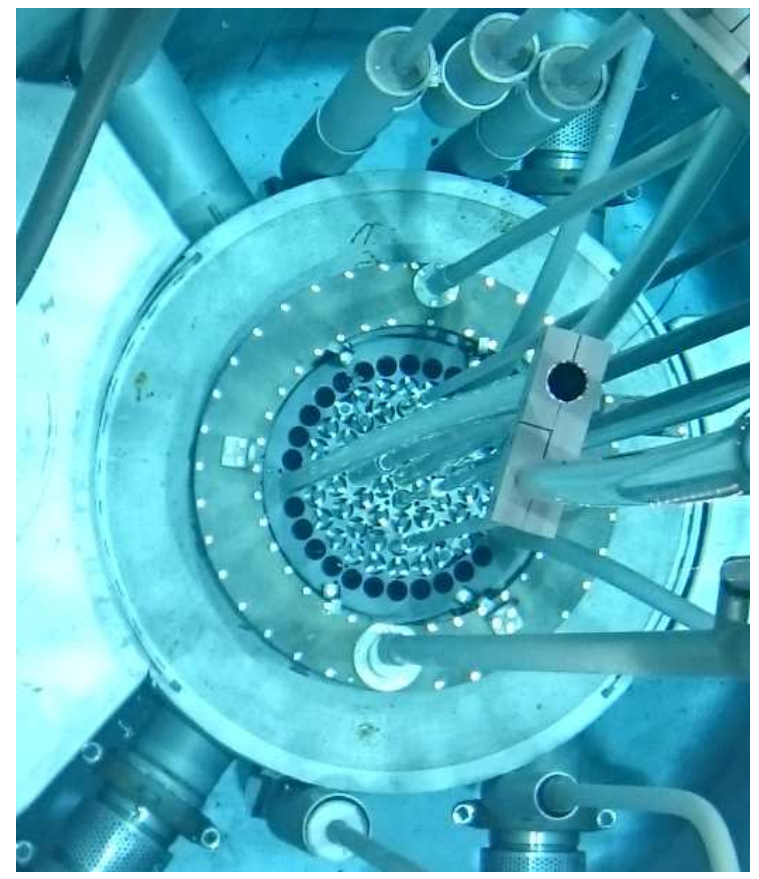

Figure 3: Underwater photograph of the TRIGA Mark II reactor core.

\section{2) Miniature gaseous detectors}

CEA-manufactured $\varnothing 3 \mathrm{~mm}$ miniature fission chambers (FC - with $10 \mu \mathrm{g}$ of ${ }^{235} \mathrm{U}$ ) with mineral integrated cable were used for MONACO v2 system reactor tests. Detectors are deployed into the reactor core by using a specially designed positioning system, composed of aluminum guide tubes and a pneumatic drive consisting of a series of valves and pistons, all controlled by a microcontroller. Integrated stainless steel cables of detectors are used for inserting and withdrawing the chambers into and out of the reactor core [7].

The MONACO v2 system prototypes were first tested in the TRIGA Mark II reactor, in late 2018, using two ${ }^{235} \mathrm{U}$ miniature FCs (FC2288 and FC2289).

\section{B. Neutron axial profiles}

FC acquisition modes are tested individually while performing neutron flux axial profiles in some of the TRIGA in-core experimental locations. FCs are withdrawn step-bystep using the pneumatic moving system; for each step, 30 seconds MONACO v2 acquisitions are performed. Figure 4 displays two profile runs: a first one at $100 \mathrm{~W}$ with the pulse mode operational and a second one at $100 \mathrm{~kW}$ with both Campbell and current modes operational.

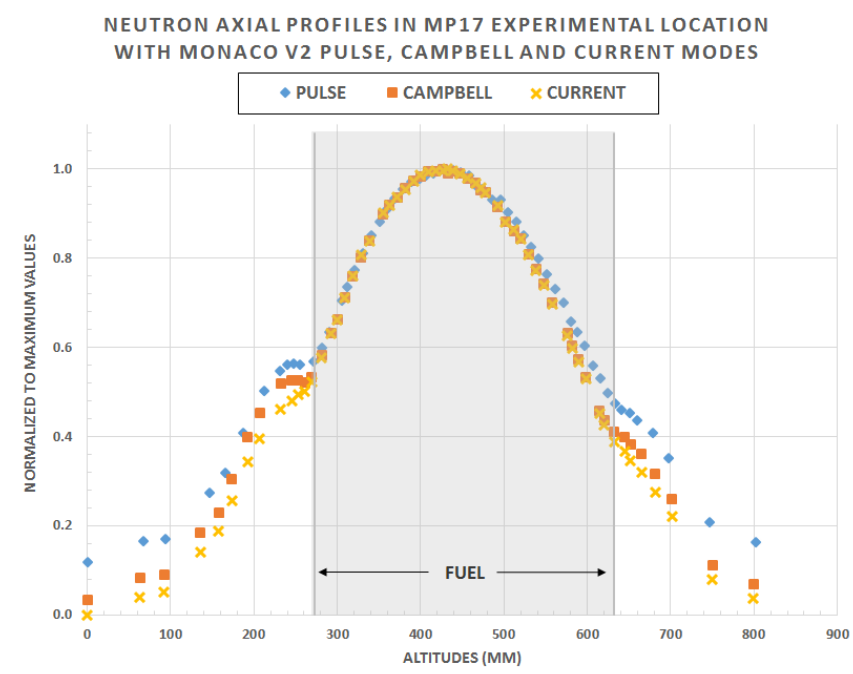

Figure 4: Normalized axial neutron flux profiles in pulse, Campbell and current modes using MONACO v2 system and FC2288.

Normalized values are describing the same neutron profile shape, validating the three modes when operated in their predestined neutron flux range. Current mode has a mixed sensitivity to neutron and gamma. Neutron/gamma contribution ratios are strongly changing at both fuel interfaces, explaining the quasi absence of signal bump in that region in current mode.

\section{Operating mode linearity and alignment}

The two FCs were also used to check the linearity of the three operating modes independently. The operators stabilized the reactor power at different power steps from $10 \mathrm{~W}$ to $250 \mathrm{~kW}$ (reactor nominal power). At each power step, MONACO v2 measurements were averaged over 60 seconds.

TABLE I. MONACO v2 LINEARITY TO REACTOR POWER STEPS (FC2288) [ITALIC AND GREY FIGURES ARE OUT OF OPERATIONAL RANGE]

\begin{tabular}{llcc} 
Power & Counts & Campbell & Current \\
\hline $10 \mathrm{~W}$ & $2.69 \mathrm{E}+03$ & $1.21 E-21$ & - \\
$100 \mathrm{~W}$ & $2.67 \mathrm{E}+04$ & $1.48 \mathrm{E}-21$ & - \\
$1 \mathrm{~kW}$ & $2.55 \mathrm{E}+05$ & $3.65 \mathrm{E}-21$ & - \\
$5 \mathrm{~kW}$ & $1.01 \mathrm{E}+06$ & $1.30 \mathrm{E}-21$ & - \\
$7 \mathrm{~kW}$ & $1.28 \mathrm{E}+06$ & $1.78 \mathrm{E}-21$ & - \\
$10 \mathrm{~kW}$ & $1.56 \mathrm{E}+06$ & $2.51 \mathrm{E}-21$ & $1.63 \mathrm{E}-08$ \\
$50 \mathrm{~kW}$ & $1.01 \mathrm{E}+06$ & $1.19 \mathrm{E}-20$ & $1.20 \mathrm{E}-06$ \\
$100 \mathrm{~kW}$ & $2.03 \mathrm{E}+05$ & $2.36 \mathrm{E}-20$ & $2.69 \mathrm{E}-06$ \\
$150 \mathrm{~kW}$ & $4.51 \mathrm{E}+04$ & $3.53 \mathrm{E}-19$ & $4.19 \mathrm{E}-06$ \\
$200 \mathrm{~kW}$ & $1.45 \mathrm{E}+04$ & $4.77 \mathrm{E}-19$ & $5.79 \mathrm{E}-06$ \\
$250 \mathrm{~kW}$ & $7.48 \mathrm{E}+03$ & $6.22 \mathrm{E}-19$ & $7.65 \mathrm{E}-06$
\end{tabular}

The three mode results are presented in Table I. Each mode considered separately is working as expected: pulse mode operational for low neutron flux range, Campbell mode for middle and high range and current mode for high range. All three modes demonstrating a good linearity to reactor power. 
Pulse mode saturates and diverges from linearity when the fission rate in the detector leads to a count rate higher than $\sim 1.5 \mathrm{E}+06$ (without any dead time correction implemented). Campbell and Current modes present specific minimum fission rate to be operational and linear to the reactor power.

Figure 5 shows the three modes normalized and aligned providing some pulse/Campbell and Campbell/current alignment coefficient. The dashed line with its associated its $\mathrm{Ch} 2$ test proves the acquisition system linearity over the three modes. Figure 5 demonstrates that MONACO v2 FC acquisition modes are available for very wide neutron flux range. The system performance test is here limited by the reactor maximum power.

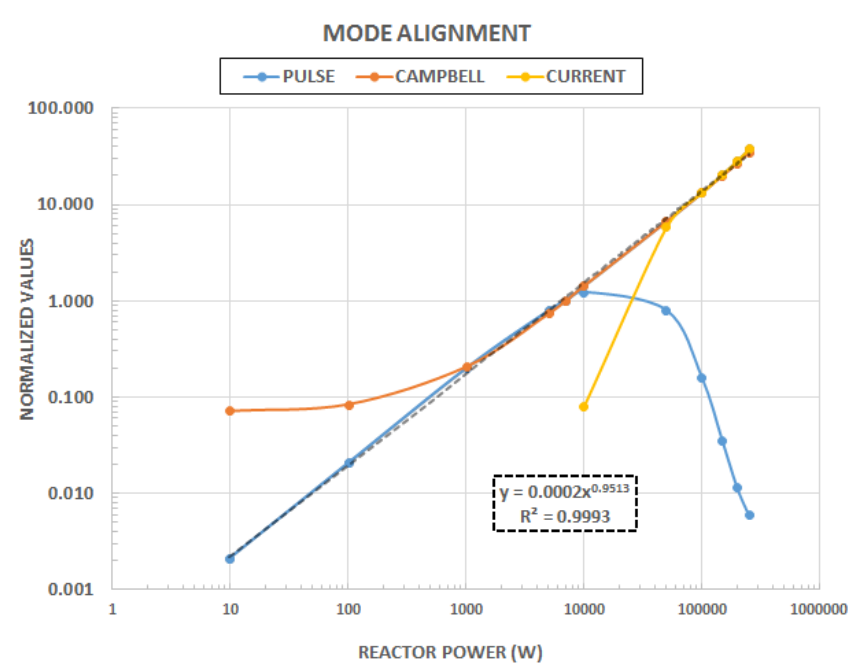

Figure 5: Normalized values of pulse, Campbell and current modes recorded during a reactor power ramp, using MONACO v2 system and FC2288. The dashed line represents the trend curve with associated Chi2 test.

Figure 5 also highlights the pulse/Campbell mode overlapping over almost one neutron flux decade.

\section{CONCLUSION AND PERSPECTIVES}

The current mode, as implemented at the time of the test, is not fulfilling the system requirements for low current range down to $\ln \mathrm{A}$, particularly regarding low current miniature ion chamber (MIC) for gamma measurements. Actual current mode method is only operational from $0.1 \mu \mathrm{A}$. New current measurement approach is under development and will be tested in the coming weeks.

Maximum neutron flux in the TRIGA reactor is limited to $\sim 2.20 \mathrm{E}+13 \mathrm{n} . \mathrm{cm}^{-2} \cdot \mathrm{s}^{-1}$ in steady state operation. This neutron flux level is not high enough to test MONACO v2 systems in whole Material Testing Reactor conditions (i.e. future Jules Horowitz Reactor - JHR - under construction at CEA Cadarache site).

To challenge the MONACO v2 system upper limits, it will be tested in 2020 during the next CEA - JSI bilateral collaboration project aiming at very high neutron flux measurements during transient power reactor pulses up to few hundreds MW. The neutron flux is expected to reach $\sim 1.00 \mathrm{E}+15 \mathrm{n} . \mathrm{cm}^{-2} \cdot \mathrm{s}^{-1}$ during $\sim 100 \mathrm{~ms}$. This will be also a unique occasion to estimate the Campbell/current mode overlapping range.

Beyond encouraging the increase of on line local neutron and gamma measurements with gaseous detectors in research reactor experiments, the MONACO v2 system may also be used in the future for self-powered neutron detectors (SPND) as its current mode working range (down to $1 \mathrm{nA}$ ) is compatible with SPND measurements.

MONACO v2 system development will shortly end and CEA is already in contact with industrial companies to make it available on the nuclear instrumentation market

\section{ACKNOWLEDGMENT}

This development was performed with funding from the Nuclear Instrumentation Project of the CEA Nuclear Energy Division through a collaboration of two CEA labs: the Instrumentation, Sensors and Dosimetry Lab (LDCI) and the Sensors and Electronics Lab (LCAE).

The work performed at the JSI TRIGA reactor was in preparation of the 2019-2021 bilateral collaboration between CEA and Ministry of higher education, science and technology of Slovenia.

Authors are grateful to the TRIGA Mark II reactor operators for their constant availability to run the reactor and the preparation of the experiments.

\section{REFERENCES}

[1] M. Thevenin, L. Barbot, G. Corre, and R. Woo, "Digital Real-Time Multiple Channel Multiple Mode Neutron Flux Estimation on FPGAbased Device," in Fourteenth International Symposium on Reactor Dosimetry, Aix-en-Provence, France, 2014.

[2] L. Barbot, C. Domergue, J.F. Villard, C. Destouches, G. Braoudakis, D. Wassink, B. Sinclair, J. Osborn, H. Wu and C. Blandin, On line neutron flux mapping in fuel coolant channels of a research reactor, in Advancements in Nuclear Instrumentation Measurement Methods and their Applications (ANIMMA), Marseille, France, 2013.

[3] Wide range neutron detection system, by W. H. Todt (1978, Aprils 25) US Patent 4086490 A [Online]. Available at http://www.google.com/patents/

[4] L. Barbot, S. Normand, P. Pasdeloup and B. Lescop, Final qualification of an industrial wide range neutron instrumentation in the OSIRIS MTR reactor. , in Advancements in Nuclear Instrumentation Measurement Methods and their Applications (ANIMMA), Marseille, France, 2009.

[5] Y. Moline, A. Dabat-Blondeau, H. Hamrita, S. Klasa, G. Corre, J-M. Bourbotte, K. Boudergui, L. Barbot, C. Destouches, MONACO: A Case Study of a New System on Chip and Stream Based Architecture for Nuclear Instrumentation, to be presented in Advancements in Nuclear Instrumentation Measurement Methods and their Applications (ANIMMA), Portorož, Slovenia, 2019.

[6] Z. Stancar, L. Snoj, L. Barbot, Reaction Rate Distribution Experiments at the Slovenian JSI TRIGA Mark II Research Reactor, TRIGA-FUNDRESR-002, International Handbook of Evaluated Reactor Physics Benchmark Experiments, Organization for Economic Cooperation and Development - Nuclear Energy Agency, NEA/NSC/SOC(2006)1, Paris, 2017.

[7] L. Barbot, C. Domergue, S. Breaud, C. Destouches, J.-F. Villard, L. Snoj, Ž. Štancar, V. Radulović, and A. Trkov, Neutron Field Characterization of Irradiation Locations Applied to the Slovenian TRIGA Reactor, in Advancements in Nuclear Instrumentation Measurement Methods and their Applications (ANIMMA), Marseille, France, 2013. 